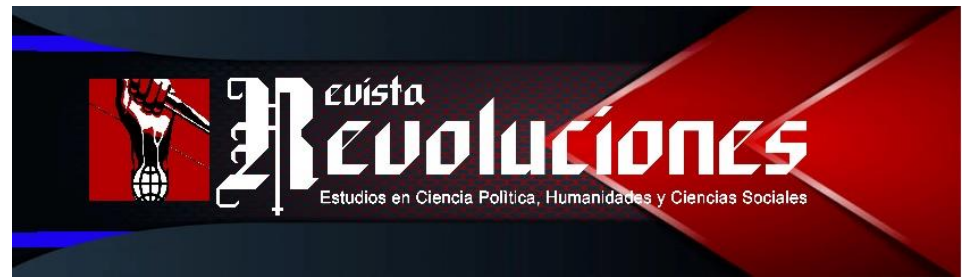

\title{
ENSAYO
}

\section{RE-SIGNIFICACIONES SOBRE TRABAJO COLABORATIVO DE ESTUDIANTES DE POSGRADO}

\author{
Re-significations on collaborative work of graduate \\ students
}

María Mellado

UNIVERSIDAD CATÓLICA DE TEMUCO CHILE

mmellado@uct.cl

http://orcid.org/oooo-ooo1-

8358-0845

Diego Queulo

COLEGIO SANTA CRUZ DE VICTORIA CHILE

Diego.queulo11@gmail.com

https://orcid.org/oooo-ooo1-

8408-1239

\author{
Juan Chaucono \\ UNIVERSIDAD CATÓLICA DE TEMUCO \\ CHILE \\ jchaucono@uct.cl \\ https://orcid.org/o000-0002- \\ 6021-7047 \\ Claudia Hormazábal \\ LICEO BICENTENARIO DE TEMUCO \\ CHILE \\ Clauhormazabal1@gmail.com \\ https://orcid.org/oooo-0oo1- \\ 8958-808X
}

\author{
Romina Melivilu \\ ESCUELA MONSEÑOR GUIDO DE RAMBERGA \\ Romina.melivilu.neveu@gmail.com \\ https://orcid.org/oooo-0002- \\ 3788-0967 \\ Amanda Arteaga \\ CORPORACIÓN EDUCATIVA ESCUELA SAN JUAN \\ CHILE \\ Agaarte.amanda@gmail.com \\ https://orcid.org/oooo-0oo1- \\ 9608-8606
}

DOI: https://doi.org/10.35622/j.rr.2021.06.006

Recibido: 30-III-2021 / Aceptado: 28-X-2021 / Actualizado: 01-XI-2021

\section{Resumen}

El presente ensayo pretende innovar en la formación de posgrado mediante las resignificaciones que van construyendo los estudiantes al iniciar el primer módulo del itinerario formativo en Gestión Escolar de una Universidad de la región de La Araucanía, Chile, a través de un método dialógico crítico y de reflexión permanente entre pares y docentes sobre las propias prácticas realizadas en su centro escolar. Observamos, que esta ruptura de representaciones implícitas sobre trabajo colaborativo requiere de una toma de consciencia del aprendiz y de una autorregulación permanente de su propia práctica como docente o directivo escolar, con foco en el aprendizaje del estudiante. La propuesta que presenta este ensayo tributa a la transición representacional que han gestado los estudiantes durante y después del primer módulo del curso Estado del Arte de la Gestión Escolar. En conclusión, se requiere profundizar en el mejoramiento de las prácticas para lograr observar, si este cambio representacional afecta de manera paralela el actuar profesional de los estudiantes de posgrado en gestión escolar. 
Palabras Clave: Aprendizaje, liderazgo escolar, trabajo colaborativo, resignificación.

\begin{abstract}
This essay aims to innovate in postgraduate training through the resignifications that students build when starting the first module of the training itinerary in school management at a university in the Region of La Araucanía, Chile through a critical dialogue method and permanent reflection between peers and teachers about the practices carried out in their school. We observe that this breakdown of implicit representations about collaborative work requires an awareness of the learner and a permanent self-regulation of their own practice as a teacher or school director with a focus on student learning. the proposal presented in this essay pays tribute to the representational transition that students have made during and after the first module of the state of the art course in school management. In conclusion, it is necessary to deepen in the improvement of practices to be able to observe, if this representational change affects in a parallel way the professional performance of graduate students in school management.
\end{abstract}

Keyword: Learning, school leadership, collaborative work, resignification.

\title{
INTRODUCCIÓN
}

En Chile desde hace un tiempo el trabajo colaborativo se ha transformado en una de las estrategias más promovidas por el Ministerio de Educación (Centro de Perfeccionamiento, Experimentación e investigaciones Pedagógicas, 2019) para favorecer la cohesión de los integrantes de la comunidad escolar con el fin de entregar una respuesta educativa acorde a las características y necesidades del estudiantado. Frente a esto, diversos autores respaldan lo mencionado y coinciden en comprender que el trabajo colaborativo es una práctica de liderazgo pedagógico que permite movilizar e intencionar para transformar la cultura escolar de los centros escolares (Calvo, 2014; Chaucono, Mellado y Yuste, 2020; García, M., García, P., Guevara, Herrera, 2015). Por lo anterior, a nivel nacional se han establecido leyes y decretos desde la política pública de educación (Ley General de Educación) para impulsarla de manera intencionada al interior de los establecimientos educacionales.

Sin embargo, a pesar de la normativa vigente, aún se desconocen los progresos significativos en la forma de abordar el trabajo en los centros escolares, configurando de este modo un problema de práctica que incide directamente en el núcleo pedagógico.

En este sentido, en la práctica docente es posible observar que el trabajo colaborativo se gestiona bajo el predominio del liderazgo administrativo, posiblemente con el afán de cumplir con los protocolos de supervisión externa, priorizando esta visión por sobre una mirada pedagógica y centrada en el aprendizaje. Conforme a la razón mencionada, un porcentaje considerable de los equipos profesionales y de aula trabajan de manera disgregada, 
respondiendo sólo a la normativa vigente, mas no con los principios emanados desde un aprender dialogado, recíproco y democrático.

Desde este escenario se hace necesario reflexionar y resignificar las concepciones de base respecto al trabajo colaborativo para generar una teoría de acción que permita transformar los modos habituales de realizar docencia. El presente ensayo es el resultado de una reflexión colectiva en el marco de estudios de Magíster en Gestión Escolar de la Universidad Católica de Temuco, Chile, guiados por docentes cuyo rol para la investigación fue de amigo crítico. En primera instancia, se indagó en las representaciones sobre trabajo colaborativo que manifestaron los participantes a la luz de una exhaustiva revisión bibliográfica que permitió resignificar dichos saberes implícitas sobre el trabajo colaborativo al incio del magíster para avanzar desde prácticas de trabajo colaborativo hacia la construcción de aprendizaje colaborativo como fuente de desarrollo profesional.

\section{METODOLOGÍA}

La metodología que utilizó este ensayo corresponde a los diálogos críticos y la reflexión permanente sobre la propia práctica docente y directiva que se desarrollaron en las diversas instancias de aprendizaje realizadas antes y durante el módulo Estado del Arte de la Gestión Escolar, cuyo propósito de estas estrategias permitieron resignificar las representaciones sobre el trabajo colaborativo que manifestaban los cuatro participantes en formación guiados por dos docentes del magíster. Lo anterior, permitió documentar que el ensayo argumentativo sobre el propio actuar docente permite dar una mejor alternativa para aprender desde la reflexión y acción del desempeño profesional docente en un contexto determinado.

\section{DESARROLLO}

El trabajo colaborativo constituye una de las principales estrategias utilizadas para la promoción de aprendizajes con una mirada inclusiva (Mellado, Chaucono, Hueche y Aravena, 2017; Rodriguez y Ossa, 2014; Stainback y Stainback, 1999). Este se comprende como una metodología que impulsa el estudio, el intercambio de experiencias y el análisis de prácticas pedagógicas entre docentes en espacios determinados (CPEIP, 2019). De este modo, distintos estudios apuntan al rol que asume este tipo de trabajo en la promoción de comunidades de aprendizaje profesional docente (en adelante, CAP), en pos del desarrollo profesional basado en una cultura de la colaboración (Bolívar, 2019; CPEIP, 2019; Fambona, Iglesias y Lozano, 2016; Durán, 2014). En otras palabras, el trabajo colaborativo se transforma en una estrategia potente para contrarrestar las representaciones implícitas sobre el aprendizaje que orientan las decisiones a la hora de liderar y gestionar el desarrollo profesional docente y aprendizaje del estudiante.

Del mismo modo, García et al., (2015) plantean que el trabajo colaborativo está basado en principios fundamentales como la reflexión conjunta y la motivación por innovar con foco en

Revista Revoluciones -68- Vol. 3, No 6 (2021), pp. 66-77

Esta obra está bajo una licencia internacional Creative Commons Atribución 4.0. 
el aprender para resignificar las prácticas docentes. En relación a esto, diferentes autores aluden a la necesidad de generar condiciones propicias que permitan promover la participación colaborativa con el propósito de impulsar decisiones de manera compartida y situadas en virtud de las necesidades y problemáticas que emergen en las salas de clases (CPEIP, 2019; Elige Educar, s.f; Seashore \& Murphy, 2019). Para esto, Leithwood, Day, Sammons, Harris y Hopkins (2006) proponen la creación de tiempos comunes de planificación, así como el establecimiento de acciones concretas para la resolución de problemas, distribución del liderazgo y, en consecuencia, una mayor implicancia de los docentes en la toma de decisiones de las escuelas. Es decir, el trabajo colaborativo como estrategia depende de múltiples factores, pero sin duda requiere de un liderazgo compartido y de decisiones que se concreten en acciones que propicien transformaciones en la gestión de los centros escolares.

En tal sentido, los resultados de diversos estudios a nivel de docencia pedagógica coinciden que el trabajo colaborativo impacta de manera positiva en el núcleo pedagógico, pues permite derribar la triada desde una perspectiva tradicional entre docente y estudiante frente al contenido de aprendizaje (Elmore, 2010; Mellado, Rincón Gallardo, Aravena y Villagra, 2020; Fullan, 2019). Por otro lado, Maldonado y Sánchez (2012) plantean que esta relación horizontal en la interacción fortalece el desarrollo de habilidades y actitudes para aprender, lo que estimula un pensamiento crítico de co-responsabilidad. En otras palabras, transitar desde la segregación hacia la inclusión del conocimiento permite promover un diálogo continuo de participación democrática, siendo estos últimos elementos cruciales para gestar una verdadera comunidad de práctica con foco en el crecimiento de todos los integrantes que componen el centro escolar.

En Chile, el trabajo colaborativo se regula en los establecimientos educacionales a partir del Decreto 170 y la Ley 20.903. Si bien los equipos directivos reconocen y contextualizan estas políticas educativas en torno a la asignación del tiempo no lectivo, al consignar en la carga horaria del profesorado el trabajo colaborativo y la preparación de la enseñanza, es evidente que no le asignan una mayor relevancia, puesto que se observa que perciben este tiempo como de libre disposición de las y los educadores, abriendo la posibilidad a la asignación de tareas emergentes o irrelevantes para el aprendizaje. Por consiguiente, a partir de esta práctica, se infiere que existe la creencia que la labor pedagógica se lleva a cabo sólo dentro de la sala de clases, restándole importancia al análisis y reflexión conjunta entre profesionales sobre qué, cómo y para qué aprenden los niños, niñas y jóvenes en las escuelas.

Por otra parte, los equipos directivos han obviado la construcción conjunta con la comunidad educativa de la visión de trabajo colaborativo, extrapolando en su práctica concepciones propias, basadas en criterios y experiencias personales, las cuales no siempre representan las expectativas de la comunidad y no permiten que esta se involucre, tanto en la conceptualización como en la puesta en práctica de la misma. Asimismo, es un hecho conocido esta carencia de objetivos comunes en los establecimientos educativos, lo cual se traduce en acciones disociadas del trabajo colaborativo, puesto que cada docente concreta 
acciones conjuntas a partir de su propia interpretación, perdiendo instancias valiosas de reflexión y aprendizaje entre pares. En tal sentido, varios autores destacan que un liderazgo escolar efectivo se concibe como el ejercicio de movilizar e influenciar a otros para lograr objetivos compartidos (Beltrán, 2014; Leithwood y Riehl, 2009; Mellado y Chaucono, 2019).

Asimismo, Weinstein (2016) afirma que este liderazgo permite desarrollar un proyecto educativo, potenciar una identidad colectiva, generar expectativas y avanzar hacia una docencia que posicione a los estudiantes como núcleo central de la tarea educativa. A pesar de los avances investigativos y desde el análisis de nuestro propio desempeño, observamos que continuamos desarrollando prácticas de liderazgo poco efectivas que aún no logran impactar en el desarrollo integral del estudiante. Lo anterior es graficado en el figura 1.

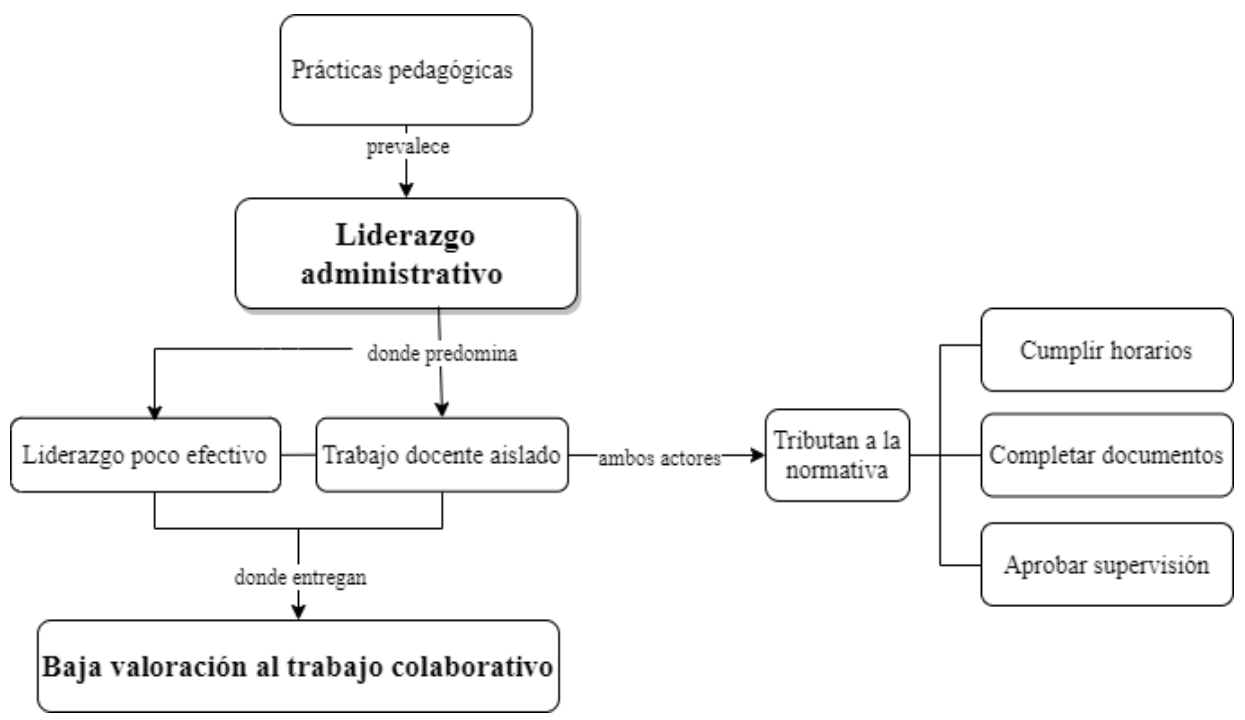

Figura 1: Problema de práctica observado desde nuestra mirada de trabajo colaborativo.

Fuente: Elaboración propia de los autores (2020).

Las prácticas de trabajo colaborativo en los establecimientos educacionales chilenos involucran directamente el tipo de liderazgo y gestión que se desarrollan en estos. Entonces, cuando están centradas en actividades administrativas, el trabajo colaborativo también se alejará del núcleo pedagógico. Por ejemplo, hoy en las escuelas, liderazgo y gestión se comprenden como conceptos diferentes, pero debieran concebirse y practicarse en total complementariedad. Si bien el primero se encarga de la realización de los cambios necesarios que ayudan a la proyección de la comunidad educativa hacia un entorno de mejora y a la construcción y alcance de objetivos comunes, el concepto de gestión, por su parte, está asociado a la creación de las condiciones organizacionales necesarias que garanticen la realización de dichos cambios, enfocados dentro de la comunidad educativa hacia las prácticas docentes y el desarrollo de las capacidades profesionales (Marco para la Buena Dirección y Liderazgo Escolar, 2015). Por consiguiente, dichos conceptos no manifiestan una diferenciación conceptual supeditada, sino más bien se relacionan entre sí de manera suplementaria, es decir, un buen líder pone al servicio del núcleo pedagógico la gestión educativa, priorizando aquellas acciones que impacten en el aprendizaje de todas y todos los estudiantes. 
En este sentido, se debe entender el concepto de liderazgo como un proceso de influencia que conduce al logro de objetivos deseados. Al respecto, Bush (2018) afirma que los líderes exitosos impulsan el desarrollo de metas comunes y se involucran en acciones conjuntas para influenciar de manera indirecta en el logro de los objetivos propuestos. De esta forma, emerge un liderazgo compartido con una configuración constitutivamente social (Bolívar, López y Murillo, 2013). En otras palabras, el liderazgo no recae en la persona, sino que se transforma en una práctica que promueve la propia cultura escolar de las escuelas, de este modo, para realizar cambios tanto en el trabajo colaborativo como en el liderazgo escolar se requiere resignificar nuestras propias representaciones sobre aprendizaje.

De este modo, las representaciones sobre aprendizaje son saberes e ideas que se construyen en la trayectoria personal en un contexto determinado que carece de rigor científico para ponerlas a prueba o en tela de juicio (Chaucono, Mellado y Yuste, 2020). Del mismo modo, diversos estudios han corroborado que las representaciones sobre aprendizaje que han construido profesionales de la educación van divorciadas de las teorías estudiadas en la formación docente (Mellado, Chaucono y Villagra, 2017; García, 2010; Pozo, Scheuer, Mateos y Puig, 2006; Pozo, 2017). En otras palabras, las representaciones implícitas sobre aprendizaje se transforman en un saber tácito distinto al conocimiento profesional que requiere ser explicitado para cuestionarlas con el fin de lograr una re-significación a la luz de los avances investigativos sobre liderazgo escolar.

\section{RESULTADOS}

En este apartado se evidenció que las representaciones de los docentes y directivos que participan del programa de Magíster en Gestión Escolar no están ajenos a los avances investigativos documentados en el estado del arte de este ensayo. Por lo tanto, se hace necesario comprender por qué a pesar de la evidencia empírica y diversas acciones impulsadas desde el Ministerio de Educación de Chile (2019) para promover prácticas de trabajo colaborativo, estas no se desarrollan de manera efectiva en las escuelas del país. A continuación, se muestra el esquema 2, que evidencia las re-significaciones que se han construido durante y posterior al Programa de Magíster en Gestión Escolar, Universidad Católica de Temuco, Chile. 


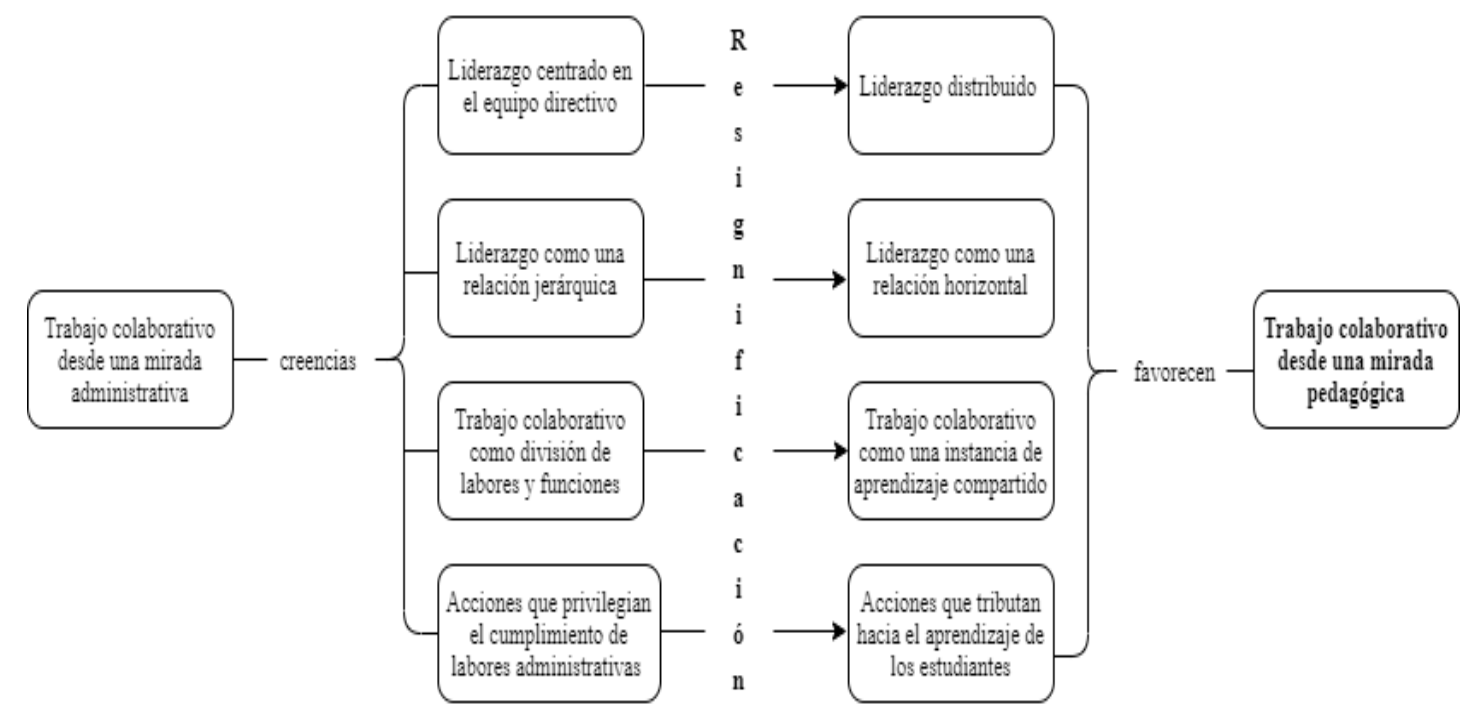

Figura 2: Resignificación de nuestra práctica docente y directiva antes y durante del módulo estado del arte de la gestión escolar.

Fuente: Elaboración propia de los autores (2020).

Como se observa en el figura 2, reflexionar acerca de nuestra propia problemática como docentes en función de los diálogos cuestionadores en clases, discusiones profesionales entre el grupo de trabajo a la luz de los avances teóricos, logró resignificar nuestras comprensiones sobre trabajo colaborativo, lo que implicó entender los paradigmas que prevalecen en una comunidad educativa. En este sentido, como actores que formamos parte de un grupo profesional en escuelas de nuestra región, debemos considerar la configuración de una visión conjunta que conduzca hacia una gestión colaborativa centrada en el liderazgo pedagógico, que permita avanzar hacia la reflexión crítica y democrática de nuestro quehacer (Bolívar, 2010; Leithtwood y Day, 2007; Mellado y Chaucono, 2016).

Estos resultados, pueden ser por las comprensiones que cada uno construye de manera individual sobre el aprender en conjunto o posiblemente por las representaciones implícitas que actúan de barrera para dar sentido a lo que se realiza en las salas de clases. No obstante, distintos estudios realizados en Chile (Mellado y Chaucono, 2016; Mellado, Chaucono y Villagra, 2017; Chaucono, Mellado y Yuste, 2020) dan cuenta que persiste en la región una mirada centrada en el profesorado asociada a la transferencia de información al estudiante, quien asimila el contenido como un receptor pasivo, obviando la interacción, horizontalidad y democracia en los diversos contextos educativos. Desde este panorama, se observa que el trabajo colaborativo requiere de una mirada común sobre "qué es aprender", "cómo se aprende" y "para que se aprende", puesto que estas comprensiones guían las prácticas a realizan en los centros escolares.

Al cambiar estos paradigmas, también se modifica la conceptualización de trabajo colaborativo y, en consecuencia, su práctica; esto involucra la puesta en acción y la responsabilidad de generar el cambio desde el plano personal. Por consiguiente, debido al reconocimiento y valoración que se le da a esta estrategia de acuerdo a su relevancia, optimizaremos en nuestra práctica el tiempo establecido para co/docencia, trabajo por 
departamentos y/o trabajo interdisciplinario, para así fijar metas a corto, mediano y largo plazo que involucren la colaboración no sólo entre docentes, sino con cualquier otro actor de la comunidad escolar, manteniendo la disposición a aprender de manera conjunta y compartida. De esta forma, buscaremos propiciar diferentes instancias que permitan, primero, develar las concepciones que se tienen a la base de las prácticas de colaboración, para luego seleccionar y construir una visión que busque representar a todos y todas sin perder el foco en el desarrollo integral del estudiante. Finalmente, es esencial asumir como estudiante de posgrado en Gestión Escolar que nuestras funciones como futuros gestores se pudiesen realizar desde una concepción de líder aprendiz que promueve el aprendizaje colectivo vinculado permanentemente al núcleo pedagógico, convirtiéndonos en promotores del cambio que involucre la permanente mejora escolar (Montesinos \& Uribe, 2016; Elmore, 2010; Rincón-Gallardo y Fullan, 2015).

\section{CONCLUSIONES}

Ante el escenario planteado a lo largo de este ensayo, es relevante considerar algunos puntos que enriquecen la discusión de la temática del trabajo colaborativo en las escuelas. En primer lugar, creemos que la dinámica de la distribución de labores en el sistema educativo es consecuencia del modelo económico imperante y de cómo este ha permeado la sociedad y con ello la educación. Ante esto, Villalobos y Quaresma (2015) argumentan que las potentes políticas de rendición de cuentas que rigen el modelo orientado al mercado imperante han traído consigo fenómenos como la segregación y la escasa libertad de elección en el sistema educativo actual; del mismo modo, han tomado prestado algunos conceptos propios de una lógica mercantilista como "competencia", "eficiencia" y "evaluación de desempeño", asignándole valor a la eficacia como fin último, lo cual deja en segundo plano aspectos vitales como la identidad de cada proyecto educativo, y la generación de espacios para la innovación y cambio en pro del logro de los aprendizajes. Por su parte, Deere \& Strayer (2001) han concluido que la lógica del sistema de incentivos tiene incidencia directa en los objetivos planteados dentro de la escuela, esto se comprende porque su subsistencia está sujeta a distintos factores, algunos ajenos al núcleo pedagógico, lo que explica por qué resulta tan sencillo para las escuelas perder el foco y desvirtuar sus acciones de mejora.

Otros autores, por su parte, han manifestado que el sistema de vouchers, el cual también existe en algunos países como Australia, Nueva Zelanda, Dinamarca, Suecia, Singapur, Corea del Sur y Chile, pese a que tiene aspectos positivos como el aseguramiento de la equidad de acceso y la libertad de elección (Gallego, 2002; Joiko, 2011), en nuestro país ha favorecido la competitividad en y entre las escuelas (Villalobos y Quaresma, 2015), así como la preferencia de los y las estudiantes y sus familias por el sistema privado (Hsieh \& Urquiola, 2006). Esto perjudica directamente el desarrollo de espacios de colaboración, debido a que esta valiosa instancia de aprendizaje no ocurre en espacios donde prima la competencia, pues es lo opuesto a ella. Sostener el culto a la eficiencia en las escuelas tiene un costo claro y es la optimización, no solo de los recursos económicos, sino también del aprendizaje. 
Desde este escenario, América Latina se encuentra recién dando los primeros pasos en lo que a investigación sobre liderazgo escolar se refiere. Así, en países como Chile y en todo el resto de la región, se ha intentado impulsar cambios, pero se tiende a producirse un completo desbalance entre las medidas de política que son cada vez más frecuentes y extendidas y su sustento en investigaciones contextualizadas sobre la realidad del liderazgo escolar, lo que amplifica el riesgo de la importación de soluciones descontextualizadas de nuestras necesidades y posibilidades (Aravena \& Hallinger, 2018; Cancino y Monrroy, 2017). Por ejemplo, en los países de mayor desarrollo social y educativo, la investigación respecto al vínculo del liderazgo y la mejora escolar es preponderante, ha ido además acompañando y contribuyendo a una, cada vez más profusa, agenda de políticas en este tema, que permitan decidir de manera más efectiva a los líderes educativos en base a la evidencia y no solo de manera intuitiva o de percepciones vagas de solo algunos momentos de los diferentes procesos del ámbito educativo.

Por consiguiente, se requiere resguardar de decisiones que avancen en la implementación de líneas de acción eficaces y centradas en el núcleo pedagógico, considerando que la generación de nuevas políticas y planes o programas de desarrollo debieran incorporar estrategias de fortalecimiento del liderazgo como uno de sus componentes principales, siendo inclusive un factor sobre el cual se pueda articular toda la política, pues en la autonomía que poseen los establecimientos en el sistema escolar, las decisiones producidas e implementadas por los propios centros educativos son claves para una mejora sostenible y que responda a las necesidades particulares de este (Cancino y Monrroy, 2017). En conclusión, la escasa evidencia, investigación y estudio de los líderes educativos en sus diferentes niveles, muchas veces, no permite que las políticas locales, proyectos, planes y programas de desarrollo profesional, como por ejemplo, los de trabajo colaborativo, comunidades de aprendizaje, interdisciplinariedad $\mathrm{u}$ otros, sean consistentes y propios de su proyecto educativo, al no conocer en profundidad estas prácticas que permiten avanzar hacia la mejora de los aprendizajes de los estudiantes, quedando solo a la improvisación y voluntad de los profesionales.

De esta forma, se demanda de un cambio en los modos habituales de realizar docencia que impulse una verdadera mirada crítica a nuestras propias prácticas pedagógicas o directivas. $\mathrm{Al}$ respecto, Fullan (2019) afirma que los cambios a nivel de escuela debiesen surgir desde las propias demandas que afloran en las prácticas de aula. Dicho de otra manera, intencionar la mejora desde nuestro marco de actuación podría impulsar desde adentro hacia una fuera un verdadero cambio que implique una mejora sustancial de nuestro quehacer profesional.

\section{REFERENCIAS BIBLIOGRÁFICAS}

Aravena F., \& Hallinger, P. (2018). Systematic review of research on educational leadership and management in Latin America, 1991-2017. Educational Management $\begin{array}{llll}\text { Administration } \quad \& \quad \text { Leadership, } & \text { 46(2), }\end{array}$ https://doi.org/10.1177/1741143217745882 
Calvo, G. (2014). Desarrollo profesional docente: el aprendizaje profesional colaborativo. Unesco-Orealc, Temas críticos para formular nuevas políticas docentes en América Latina y el Caribe: el debate actual. https://unesdoc.unesco.org/ark:/48223/pfoooo232822_spa

Cancino, V., y Monrroy, L. (2017). Políticas educativas de fortalecimiento del liderazgo directivo: desafíos para Chile en un análisis comparado con países OCDE. Ensaio: aval.pol.públ.Educ. [online]. 2017, vol.25, n.94, pp.26-58. ISSN 1809-4465. https://doi.org/10.1590/s0104-40362017000100002.

Beltrán, J. (2014). Factores que dificultan la gestión pedagógica curricular de los jefes de Unidades Técnico Pedagógicas. Revista mexicana de investigación educativa, 19(62), 939-961. Recuperado en 03 de febrero de 2021, de http://www.scielo.org.mx/scielo.php?script=sci_arttext\&pid=S1405$66662014000300014 \& \operatorname{lng}=$ es\&tlng=es.

Bolívar, A. (2010). El liderazgo educativo y su papel en la mejora: Una revisión actual de sus posibilidades y limitaciones. Psicoperpectivas, 9(2), 9-33. https://scielo.conicyt.cl/scielo.php?pid=so71869242010000200002\&script=sci_arttext

Bolívar, A., López, J. y Murillo, F. (2013). Liderazgo en las instituciones educativa: Una revisión de líneas de investigación. Revista Fuentes, 14, pp.15-60.

Bolívar, A. (2019). Una dirección escolar con capacidad de liderazgo pedagógico. Madrid. La Muralla.

Bush, T. (2018), "Professional Learning Communities and School Leadership: Empowering teachers", Educational.

Centro de Perfeccionamiento, Experimentación e Investigaciones Pedagógicas (2019). Trabajo colaborativo y Desarrollo Profesional Docente en la escuela. https://www.cpeip.cl/wp-content/uploads/2019/o3/trabajocolaborativo_marzo2019.pdf

Chaucono, J., Mellado, M., y Yuste, R. (2020). Líderes escolares: representaciones implícitas sobre aprendizaje. Revista ESPACIOS, 41(18).

Congreso de la República de Chile (2016, 04 de marzo). Crea el sistema de desarrollo profesional docente $\mathrm{y}$ modifica otras normas. Diario Oficial 41.966. http://bcn.cl/2f72c

Deere, D. \& Strayer, W. (2001). "Closing the gap: School incentives and minority test scores in Texas." Department of Economics, Texas A\&M University.

Duran, D. (2014). Aprenseñar. Evidencias e implicaciones educativas de aprender enseñando. Madrid: Narcea.

Elige educar (s.f). Trabajo colaborativo entre docentes. Ideas Docentes. http://www.ideasdocentes.cl/wp-content/files_mf/1513564317id_tarjetas.pdf

Elmore, R. (2010). Mejorando la escuela desde la sala de clases. Chile. Fundación Chile.

Fombona, J., Iglesias, M \& Lozano, I. (2016). El trabajo colaborativo en la educación superior: una competencia profesional para los futuros docentes. educação \& sociedade, 37(135),519-538. [fecha de consulta 3 de febrero de 2021]. issn: 0101-7330. disponible en: https://www.redalyc.org/articulo.oa?id=873/87346871012. 
Fullan, M. (2019). Liderar los aprendizajes: acciones concretas en pos de la mejora escolar. Revista electrónica de educación. n. 1, p. 58-65, ISSN 1982-7199 | DOI: http://dx.doi.org/10.14244/198271993074

Gallego, F. (2002). Competencia y resultados educativos: Teoría de la evidencia para Chile. Cuadernos de economía, 39(118), 309-352. https://dx.doi.org/10.4067/So71768212002011800003

García, M. (2010). Efecto de la formación inicial docente sobre la transformación de las creencias epistemológicas y de las creencias acerca de la buena enseñanza, de los estudiantes de pedagogía de la Pontificia Universidad Católica de Chile (Tesis de maestría). Pontificia Universidad Católica de Chile. http://repositorio.conicyt.cl/handle/10533/179677

García, M., García, P., Guevara, G. y Herrera, J. (2015). El trabajo colaborativo y su influencia en el desarrollo de la cultura profesional docente. Revista Gaceta Médica Espirituana, 17 (Supl. 1) http://scielo.sld.cu/scielo.php?script=sci_arttext\&pid=S160889212015000100006

Hsieh, C. T., \& Urquiola, M. (2006). Los efectos de la elección escolar generalizada sobre el rendimiento y la estratificación: evidencia del programa de cupones de Chile. Journal of public $\quad$ Economics, $\quad 90(8-9), \quad 1477-1503$. https://www.sciencedirect.com/science/article/abs/pii/So047272705001672

Joiko, S. (2011). La política de equidad y el nuevo sistema de vouchers en Chile: reflexiones críticas. Revista mexicana de investigación educativa, 16(50), 829-852. http://www.scielo.org.mx/scielo.php?script=sci_arttext\&pid=S140566662011000300008\&lng=es\&tlng=es.

Leithwood, K.; Day, C.; Sammons, P.; Harris, A. y Hopkins, D. (2006). Successful School Leadership. What it Is and How it Influences Pupil Learning. Nottingham: National College for School Leadership. University of Nottingham.

Leithwood, K. y Day, C. (2007). What we learned: A broad view [Lo que aprendimos: Una visión amplia]. En C. Day y K. Leithwood (Eds.), Successful Principal Leadershirp in Times of Change: An Internacional Perspective [Liderazgo principal éxito en tiempos de cambio] (pp. 189- 203). Springer.

Leithwood., K. \& Riehl, C. (2009). ¿Qué sabemos sobre liderazgo educativo? En K. Leithwood (Ed.) ¿Cómo liderar nuestras escuelas? Aportes desde la investigación (pp. 17-33). Chile. Fundación Chile.

Ley General de Educación $\mathrm{N}^{\circ}$ 20.370. Texto refundido, coordinado y sistematizado de la ley, con las normas no derogadas del decreto con fuerza de ley no 1, de 2005 Recuperado el 20 diciembre de 2014, de http://bcn.cl/1lz6u

Maldonado, M. y Sánchez, T. (2012). Trabajo colaborativo en el aula: experiencias desde la formación docente. Revista educare, 16(2), 93-118. https://goo.gl/iUoH6h

Mellado, M. y Chaucono, J. (2016). Liderazgo pedagógico para reestructurar creencias docentes y mejorar prácticas de aula en contexto mapuche. Revista Electrónica Educare, 20(1), 1-18. doi: http://dx.doi.org/10.15359/ree.20-1.18 
Mellado, M., Chaucono, J., Hueche, M., y Aravena, O. (2017). Percepciones sobre la educación inclusiva del profesorado de una escuela con Programa de Integración Escolar. Revista Educación, 41(1), 119-132.

Mellado, M., Chaucono, J y Villagra, C. (2017). Creencias de directivos escolares: implicancias en el liderazgo pedagógico. Psicología Escolar e Educacional, 21(3), 541-548. https://www.scielo.br/pdf/pee/v21n3/2175-3539-pee-21-03-541.pdf

Mellado, M., y Chaucono, J. (2019). Prácticas de liderazgo de docentes y directivos que favorecen el aprendizaje de estudiantes en contexto mapuche. Sophia Austral, (24), 63-81. https://dx.doi.org/10.4067/So719-56052019000200063

Mellado, M., Rincón-Gallardo, S., Aravena, O., y Villagra, C. (2020). Acompañamiento a redes de líderes escolares para su transformación en comunidades profesionales de aprendizaje. Perfiles https://doi.org/10.22201/iisue.24486167e.2020.169.59363

Ministerio de Educación, República de Chile. (2015). Marco para la Buena Dirección y el Liderazgo Escolar. Santiago de Chile: Centro de Perfeccionamiento, Experimentación e Investigaciones Pedagógicas.

Montecinos, C., \& Uribe, M. (2016). Desarrollo de Liderazgos para el Aprendizaje en el Siglo XXI, un Enfoque Sistémico. ÍNDICE, 4.

Pozo, J., Scheuer N., Mateos M. y Puig Pérez Echeverría (2006), "Las teorías implícitas sobre el aprendizaje y enseñanza”, en Juan Ignacio Pozo, Nora Scheur, Puig Pérez Echeverría, Mar Mateos, Elena Martín y María de la Cruz (eds). Nuevas formas de pensar la enseñanza y el aprendizaje, Graó.

Presidencia de la República (2010, 21 de abril). Fija normas para determinar los alumnos con necesidades educativas especiales que serán beneficiarios de las subvenciones para educación especial. Diario Oficial No 20201. http://bcn.cl/2im60

Rincón Gallardo, S. y Fullan, M. (2015). Física social del cambio educativo. Red de Escuelas Líderes

Rodríguez, F. y Ossa, C. (2014). Valoración del trabajo colaborativo entre profesores de escuelas básicas de Tomé, Chile. Estudios pedagógicos (Valdivia), 40(2), 303-319.

Seashore, K. \& Murphy, J. (2019). El enfoque del liderazgo positivo y su relevancia para las relaciones en la escuela. En Liderazgo en escuelas de alta complejidad sociocultural: Diez miradas (pp. 153-191). Universidad Diego Portales.

Stainback, S., \& Stainback, W. (1999). Aulas inclusivas: un nuevo modo de enfocar y vivir el currículo. España. Narcea.

Villalobos, C., y Quaresma, M. (2015). Sistema escolar chileno: características y consecuencias de un modelo orientado al mercado. Convergencia, 22(69), 63-84. http://www.scielo.org.mx/scielo.php?script=sci_arttext\&pid=S140514352015000300063\&lng=es\&tlng=es.

Pozo, J. I. (2017). Aprender más allá del cuerpo: de las representaciones encarnadas al explicitación mediada por representaciones externas. Infancia y Aprendizaje. Journal for the Study of Education and Development, 4O(2), 219-276.

Weinstein, J. (2016). Liderazgo educativo en la escuela. Nueve miradas. Chile. Ediciones Universidad Diego Portales-CEDLE. 